\title{
Numerical simulation of centrifugal serpentine micromixers and analyzing mixing quality parameters
}

\author{
Amir Shamloo ${ }^{1,2}$, Masoud Madadelahi ${ }^{1, *}$, Ali Akbari ${ }^{1, *}$ \\ ${ }^{1}$ Department of Mechanical Engineering, Sharif University of Technology \\ ${ }^{2}$ Corresponding author: Dr. A. Shamloo, Department of Mechanical Engineering, Sharif \\ University of Technology, Azadi Ave., Tehran, Iran, Email: shamloo@sharif.edu, \\ Tel:98-21-66165691, Fax: 98-21-66165599 \\ * Equal Contribution
}

\begin{abstract}
Centrifugal microfluidics or the Lab on a CD (LOCD) has developed vast applications in biomedical researches and analyses. Fluid mixing is an application of the LOCD. In this paper, multiple centrifugal micromixers were simulated. Various parameters were originally presumed to have an effect on mixing performance. These parameters include inlet angle, angular velocity, cross-sectional profile, perpendicular length ratio and the number of channels in series. They were each analyzed through simulations. It was gathered that the inlet angle does not significantly affect the mixing quality. Increasing angular velocity steadily increases mixing quality for all geometries. The vertical triangular cross section gives the best mixing quality and the horizontal rectangular cross section has the worst. Also both increasing the perpendicular length ratio and adding an additional microchannel in series to the original, enhances mixing.
\end{abstract}

Keywords Centrifugal, Microfluidics, Micromixer, Mixing Parameters, Mixing Quality

\section{1-Introduction}

The Lab-On-A-Chip (LOC) technology has been widely used in several biomedical and chemical analyses such as the point-of-care testing (POCT) (Figeys and Pinto [1], Haeberle, et al. [2], Chin, et al. [3], Ryu, et al. [4], Lee and Choi [5]). Comparing to other experimental platforms, the LOC has an advantage due to miniaturization, I.E. the consumption of both the sample and the reagent is drastically reduced (Srinivasan, et al. [6], Fair [7]). Multiple modules could be built on the LOC for different purposes in biomedical diagnostics. However, in the LOC each module requires a separate micropump to operate. Implementing several micropumps in the LOC, complexes the design and thus multifunctional LOCs are rarely used. The LOCD is a type of LOC built on a CD-shaped disk which does not require any micropumps and could operate 
with a single rotational motor. The disk is mounted on a rotor shaft and its angular motion creates centrifugal force, which is used as the substitute for the micropump in order to operate different modules on the LOCD. The convenience of operation of the LOCD has made it a favorable platform for POCT and biomedical analyses such as biochemical studies (Lee, et al. [8]), cell lysis and enzymatic studies (Kim, et al. [9], Kim, et al. [10], Lai, et al. [11]) and DNA analysis (Jia, et al. [12]). In order to conduct such studies and analyses, microfluidic functions such as micro valves and micromixers are developed on the LOCD (Madou, et al. [13], Noroozi, et al. [14], Chakraborty [15]).

For any chemical reaction to be efficient, a proper mixing of the reagents is essential. Appropriate solutions exist for mixing fluids in large scales. However, in the microfluidic scale, an effective mixing of two fluids still remains a challenge as of now. Since in microfluidic systems the flows are mostly laminar and the Reynolds number is rather small, the turbulent mixing of two fluids due to turbulence vortices would not occur. Micromixers generally fall within two categories; active micromixers which use an external force field to enhance mixing such as magnetic fields (Wang, et al. [16]) or electrical fields (Wu and Liu [17]) and passive micromixers which only rely on the geometric design of the LOCD (Tofteberg, et al. [18],Hessel, et al. [19]).

Stroock, et al. [20] developed a passive pressure-driven micromixer for mixing fluids at low Reynolds numbers. They emplaced special obstacles on the floor of the channel such that flowing over them would enhance mixing. Several micromixers have been developed for the LOCD platform. Existing micromixers mostly lack sufficient mixing performance in spite of their rather complex designs. Haeberle, et al. [2] proposed a centrifugal micromixer implemented on a straight radial microchannel which worked based on the transverse flow caused by the Coriolis force. Although their design was the simplest centrifugal micromixer, its mixing performance was limited since it strongly depended on the angular velocity of the disk and its resulting Coriolis force. Grumann, et al. [21] achieved batch-mode mixing in an LOCD with use of magnetic particles which were distributed within the two not-yet-mixed fluids. Noroozi et al. 2009[14] presented a reciprocating micromixer composed of two reservoirs connected by Ushaped microchannels. Due to geometrical limitations, application of this micromixer in an integrated LOCD platform is problematic, despite its high mixing performance. Duffy, et al. [22], Zoval and Madou [23] and Puckett, et al. [24] used a serpentine microchannel system implemented on an LOCD for enzymatic analyses, bacterial analyses and protein-ligand binding analyses; respectively. They used the serpentine design only to lengthen the flow path in order to increase the diffusion time to ensure sufficient mixing without having a detailed observation of the mixing process.

The main required characteristic of an LOCD for being used in POCT diagnostics is disposability. Thus, the fabrication of the LOCD should be cost-effective and easy. Therefore, as long as an appropriate mixing performance is ensured, a simple passive design without any additional components such as electrodes or magnets is preferable to a rather complex active design. A simple passive design is convenient to manufacture on a mass-production scale for fabrication purposes and is also portable, cost-effective and easy to use. La, et al. [25] designed and fabricated a centrifugal serpentine micromixer (CSM) on an LOCD and compared it to an original pressure-driven serpentine micromixer (PSM). Mixing in the PSM was limited since two 
laminar flows within the microchannel could only be mixed through diffusion and an inertial stirring effect happening within the sharp corners of the microchannel. However, the CSM showed superior mixing performance due to an additional secondary flow.

Finding a direct method for the quantification of mixing performance is utmostly desirable. However, this cannot be simply done, since there are quite a large number of micromixers available and yet a standard criterion to determine mixing performance does not exist (Falk and Commenge [26]). Hence, during the past couple of years, several studies have been conducted on different micromixers aiming to characterize mixer performance. Employing experimental techniques, such as fluorescent microscopy and special chemical reactions were reported in the aforementioned studies (Ehrfeld, et al. [27], Falk and Commenge [26]).These methods, allow for qualitative comparisons of micromixers. However, they do not present quantitative data, such as mixing times or mixing lengths (Aubin, et al. [28]).

Various methods previously employed for assessing the mixing performance of macro-scale static mixers, were used for micromixers. One method uses a Lagrangian analysis to follow the mixing of two fluid streams (Zalc, et al. [29]). The mixture's homogenity could be quantified via a statistical analysis of the concentration within the mixture at any section of the micromixer. This analysis is driven from Danckwerts' intensity of segregation concept which is based on the concetration's variance $\left(\sigma_{c}^{2}\right)$ at different locations with respect to the mean concentration $\left(\mathrm{c}_{\mathrm{avg}}\right)($ Danckwerts $[30])$. The mixing quality term (M.Q.) used in this paper is driven from this concept as is shown in Eq. (1). For equal volumetric feeding rates, the value of 0 for the M.Q. indicates zero mixing where a value of 1 indicates a perfect mixture.

M. Q. $=1-\mathrm{CoV}$

$\mathrm{CoV}=\frac{\sqrt{\frac{\sum\left(\mathrm{c}_{\mathrm{i}}-\mathrm{c}_{\mathrm{avg}}\right)^{2}}{\mathrm{n}-1}}}{\mathrm{c}_{\mathrm{avg}}}$

Where $\mathrm{CoV}$ is the coefficient of variation and $\mathrm{n}$ is the number of data points on which concentration was calculated. The $\mathrm{CoV}$ was analyzed at the end of each microchannel after each simulation. The mean concentration $\mathrm{c}_{\text {avg }}$ was calculated via a mass-flow weighted averaging over the concentration profile at the microchannel's outlet.

Alongside the M.Q., the dimensionless Reynolds and Peclet numbers could be used to characterize a micromixer's operation (Malecha, et al. [31]). The Reynolds number, which characterizes the flow, is defined as follows:

$\operatorname{Re}=\frac{\rho \mathrm{u}_{\mathrm{avg}} \mathrm{D}_{\mathrm{h}}}{\mu}$

Where $\rho$ is the fluid density $\left(\mathrm{kg} / \mathrm{m}^{3}\right)$, $u_{\text {avg }}$ is the mean velocity $(\mathrm{m} / \mathrm{s}), D_{h}$ is the channel's hydraulic diameter (m), and $\mu$ is the dynamic viscosity (Pa.s). The Peclet number characterizes the ratio between the mass transport due to convection and that of diffusion and is defined as Eq.(4) in which, D is the diffusivity constant $\left(\mathrm{m}^{2} / \mathrm{s}\right)$. At high Peclet numbers, the mixing is dominated by convection. 
$P e=\frac{u_{\mathrm{avg}} D_{h}}{D}$

In this paper, the mixing performance of a centrifugal micromixer is investigated through various setups. A schematic of the design is displayed in Fig.1. A serpentine microchannel is placed on a rotating disc. The center of rotation and parametric dimensions of the microchannel are depicted in Fig.1. The various setups of the problem include different values for inlet angle ( $\alpha$ ), angular velocity $(\omega)$, cross-sectional profile (rectangular, triangular and elliptic), perpendicular length ratio (b/c) and the number of channels in series. Then, M.Q. is calculated for each setup.

\section{2-Governing Equations}

The equations governing the physics of this problem are the incompressible, steady-state NavierStoke's momentum equations (Eq. (5)) along with the continuity equation (Eq. (6)) for the fluid flow and the steady-state, constant diffusivity convection-diffusion equation (Eq.(7)) for concentration distribution, all in three dimensions.

$\rho(\overrightarrow{\mathrm{u}} \cdot \nabla \overrightarrow{\mathrm{u}})=-\nabla \mathrm{P}+\mu \nabla^{2} \overrightarrow{\mathrm{u}}+\overrightarrow{\mathrm{f}}_{\mathrm{b}}$

$\nabla \cdot \overrightarrow{\mathrm{u}}=0$

$\overrightarrow{\mathrm{u}} \cdot \nabla \mathrm{c}=\mathrm{D} \nabla^{2} \mathrm{c}$

Where $P$ is the pressure $(\mathrm{Pa}), \mathrm{u}$ is the velocity vector $(\mathrm{m} / \mathrm{s}), \mathrm{c}$ is the concentration $\left(\mathrm{mol} / \mathrm{m}^{3}\right)$ and $\mathrm{f}_{\mathrm{b}}$ is the volumetric force vector which is the sum of the centrifugal and the Coriolis forces generated by the angular rotation of the disk as follows:

$\overrightarrow{\mathrm{f}}_{\mathrm{b}}=-\rho \vec{\omega} \times(\vec{\omega} \times \overrightarrow{\mathrm{r}})-2 \rho \vec{\omega} \times \overrightarrow{\mathrm{u}}$

For the fluid flow, the wall's no-slip boundary condition was applied. In the two inlets and the outlet, a constant atmospheric pressure was maintained. Water was selected as the working fluid. For the mass transfer, the no-mass flux through the walls condition was applied. At the two inlets, constant concentrations of 0 and 1 were applied. In order to ensure the diffusion of biofluids, a diffusivity constant of $6.9 \mathrm{e}-11 \mathrm{~m}^{2} / \mathrm{s}$ was selected which is for the diffusion of blood in aqueous solutions.

\section{3-Simulation}

All above equations were solved numerically through employing finite element method using COMSOL Multiphysics software. Mixing process was studied for different setups of the problem. Fig. 2 shows the different aspect ratios for rectangular cross sections.

Simulations were run for 4 different angular velocities ranging from $50 \mathrm{rad} / \mathrm{s}$ to $200 \mathrm{rad} / \mathrm{s}$ with a step of $50 \mathrm{rad} / \mathrm{s}$. Considering three different channel types (single channel, dual parallel channels and dual anti-parallel channels), simulations were run for rectangular cross-sections with three different aspect ratios (horizontal:2.56, square:1, vertical:0.39). Fig.3 displays the three different channel types used. Moreover, circular elliptic, vertical elliptic, horizontal elliptic, equilateral triangular, vertical triangular and horizontal triangular cross sections were studied. Also the effects of different values of $\mathrm{b} / \mathrm{c}$ and inlet angle on the mixing quality were investigated. 


\section{4-Results and Discussion}

The simulation results were validated by specifically running an identical simulation of the work done by La, et al. [25]. In order to do so, the geometry was extracted from La's experimental setup. Then a structured uniform mesh was built and after running several simulations, mesh independency was ensured. The problem's computational domain was discretized using 607376 three-dimensional volume elements. Fig. 4 depicts the selected mesh with two magnified regions. The total number of structured grids employed in this work's simulations varied from 200,000 to over a million, depending on the selected structured mesh and the geometric size of the problem.

In the next step of validation, the same governing equations and boundary conditions used in all other simulations were maintained for the simulation physics and the simulation was run. Fig.5(a) displays the concentration contour over the entire geometry of the validation problem. To quantitatively compare the obtained results with those of La, et al. [25], the M.Q. was calculated for various cross sections of the simulation along the channel length. Then M.Q. versus downchannel length was plotted and compared with their counterpart values of $\mathrm{La}$, et al. [25]. This can be seen in Fig.5(b). For a better understanding of the agreement between simulation results and $\mathrm{La}$, et al. [25] experiment, a curve with an $\mathrm{R}^{2}$ value of 0.99 was fit to the simulation results as Eq.(9). As it can be noticed, the obtained simulation results are in good agreement with experimental results, such that almost all of our data points fall within the error bars of La, et al. [25] results. This guarantees the physical propriety of this work.

M. $Q .=-0.7708 \exp (-0.1666 \mathrm{x})+0.8806$

The effect of perpendicular length ratio (b/c) was studied by varying different ratios of b/c. In this step a constant angular velocity of $200 \mathrm{rad} / \mathrm{s}$ was maintained within a single channel structure. Fig.6 represents M.Q. versus different perpendicular lengths. Values of volume flowrate, mean fluid velocity, Reynolds and Péclet numbers were calculated for each value of $b / c$ and are shown in Table.1. It is observed that increasing b/c, decreases the value of Pe. According to the definition of Pe (Eq. (2)), this means that the diffusion is improved. This enhances the mixing since it lengthens the diffusion time and allows the fluid agents to be mixed properly along the channel length. Although the mixing quality improves with increasing b/c, Fig.6 shows that $\mathrm{b} / \mathrm{c}$ values larger than 6 do not cause a significant improvement in mixing quality.

Table.1 Calculated values of volume flowrate, mean fluid velocity, Reynolds and Péclet numbers

\begin{tabular}{|c|c|c|c|c|}
\hline $\mathrm{b} / \mathrm{c}$ & $\mathrm{Q}(\mu \mathrm{lit} / \mathrm{s})$ & $\mathrm{U}(\mathrm{m} / \mathrm{s})$ & $\mathrm{Re}$ & $\mathrm{Pe}$ \\
\hline 1 & 12.3956 & 0.30989 & 61.978 & 898231.9 \\
\hline 2 & 10.7136 & 0.26784 & 53.568 & 776347.8 \\
\hline 3 & 9.2268 & 0.23067 & 46.134 & 668608.7 \\
\hline 4 & 8.1292 & 0.20323 & 40.646 & 589072.5 \\
\hline 5 & 7.2368 & 0.18092 & 36.184 & 524405.8 \\
\hline 6 & 6.5004 & 0.16251 & 32.502 & 471043.5 \\
\hline 7 & 5.8868 & 0.14717 & 29.434 & 426579.7 \\
\hline 8 & 5.3264 & 0.13316 & 26.632 & 385971.0 \\
\hline
\end{tabular}




\begin{tabular}{|c|c|c|c|c|}
\hline 9 & 4.8912 & 0.12228 & 24.456 & 354434.8 \\
\hline 10 & 4.4920 & 0.11230 & 22.460 & 325507.2 \\
\hline
\end{tabular}

Fig.7 and Fig.8 show the velocity and concentration contours plotted for vertical rectangular cross-sections in dual parallel channels and dual anti-parallel channels. Angular velocity was maintained at $200 \mathrm{rad} / \mathrm{s}$. As is displayed in the figures below, a secondary flow perpendicular to the main flow in the channel's axial direction has occurred. This secondary flow is created due to the Dean Flow effect. When the Reynolds number is small, the viscous forces are dominant and inertial effects are neglected, such that often the Stokes flow approximation is assumed to solve the governing Navier-Stokes equations. However, when the Reynolds number is relatively large, as is shown in Table.1, the inertial forces can no longer be neglected and inertial effects such as the Dean flow arise. The Dean Flow effect is the creation of secondary vortices transverse to the fluid flow direction as the fluid flows through a curved bend. The r-direction Navier-Stokes equation requires the pressure to increase with increasing radial distance as follows (Kundu and Cohen [32]):

$\frac{\partial \mathrm{P}}{\partial \mathrm{r}}=\frac{\rho \mathrm{u}_{\theta}^{2}}{\mathrm{r}}$

Where $r$ is the radial distance from the center of curvature and $\mathrm{u}_{\theta}$ is the tangential velocity. Therefore, the pressure is higher in regions further from the center of curvature, I.E., regions near the outer channel wall have a higher value of pressure than those near the inner channel wall. This results in a pressure gradient which pushes the fluid elements towards the inner wall. In central regions far from the wall's boundary layer, the velocity is relatively large and the centripetal pressure gradient cannot push the flow towards the inner wall. However, in peripheral regions in the vicinity of the wall's boundary layer, the velocity is rather small and the centripetal pressure gradient pushes the flow towards the inner wall. This causes a rotary secondary flow in form of a vortex. Subsequently, the cross sectional streamlines form two tangential vortices with opposite directions, one above the channel's middle plane and another below it. Hence, mixing is enhanced as the secondary flow stirs the fluid flow.

Fig.9 to Fig.11 show the mixing quality versus angular velocity of 50, 100, 150, 200 (rad/s) for single, dual parallel and dual anti-parallel channel structures; respectively. The corresponding Reynolds number for each value of angular velocity is presented above the figure according to the figure's legend. It is noticed that in all plots, M.Q. increases with increasing angular velocity. The reason is that the more the angular velocity, the more the Coriolis acceleration, which produces secondary flows, as discussed before. The secondary flow plays an important role in mixing of the fluids. Also it is noted that dual channels show much better mixing than the single channel. This is due to the fact that an increase in the microchannel length increases diffusion time which results in improved mixing qualities. The parallel and the anti-parallel channels almost show the same behavior. As for the rectangular cross sections, the square cross section has resulted in the best M.Q. in almost all simulations and then the vertical cross section comes second. The horizontal cross section does not generally yield good mixing qualities since it cannot develop an appropriate secondary flow. Although the vertical cross section develops a 
stronger secondary flow, the square cross section has resulted in a better M.Q. due to less geometrical limitations.

The effect of the channel inlet angle, I.E. the angle between the two rectangular inlets was studied on the M.Q.. In this step, a constant angular velocity of $200 \mathrm{rad} / \mathrm{s}$ was considered in a single channel setup. Fig.12 depicts M.Q. for various inlet angles. As it can be seen, $\alpha$ does not have a significant effect on the M.Q. and its minor effects could almost be neglected. It is because different inlet angles can only change the fluid's initial velocity and do not contribute to forming a secondary flow which plays the main role in the mixing process.

Other cross-sectional profiles with the same cross-sectional area were studied including three elliptic cross sections and three triangular cross sections. The aspect ratios used for these cross sections were the same as those used in rectangular cross sections. As is observed in Table.2, the best M.Q. has occurred in the vertical triangular cross section and the worst M.Q. has occurred in the horizontal rectangular cross section. The mean value for M.Q. in a cross-sectional profile was calculated $41.4 \%$ for rectangular profile, $67.2 \%$ for elliptic profile and $69.2 \%$ for triangular profile. Therefore, the triangular profile is nominated for best mixing performance. All data in Table. 2 pertain to single channel structures with an angular velocity of $200 \mathrm{rad} / \mathrm{s}$.

Table.2 M.Q. for different cross-sections

\begin{tabular}{|c|c|c|}
\hline Cross-sectional Profile & Aspect Ratio & M.Q. (\%) \\
\hline Vertical Elliptic & 0.39 & 69.2 \\
\hline Circular Elliptic & 1 & 74.9 \\
\hline Horizontal Elliptic & 2.56 & 57.5 \\
\hline Vertical Triangular & 0.39 & 78.2 \\
\hline Equilateral Triangular & 1 & 76.7 \\
\hline Horizontal Triangular & 2.56 & 52.8 \\
\hline Vertical Rectangular & 0.39 & 37.2 \\
\hline Square Rectangular & 1 & 52.5 \\
\hline Horizontal Rectangular & 2.56 & 34.6 \\
\hline
\end{tabular}

In Fig.13 to Fig.15, the concentration contours are plotted for different cross-sectional profiles with their own different aspect ratios. Both whole-channel and middle-cross sectional concentration contours are plotted. Also, the velocity field is displayed in these contours with arrows. The two tangential vortices are clearly noticed which cause a stirring effect through the secondary flow caused by the Coriolis force. The secondary flow is best created in cross sections with smaller aspect ratios. The velocity/concentration contours plotted for different crosssectional profiles show that in horizontal cross sections, the secondary flow cannot fully form. In this case, the streamlines tend to form converging vortices but cannot meet. However, in the square cross sections and the vertical cross sections, the secondary flow clearly occurs which enhances mixing through a stirring effect. Thus, explaining the lower mixing qualities for horizontal cross sections in Fig.9 to Fig.11. This stands right not only for rectangular profiles, but also for triangular and elliptic profiles. 
In order to reach an optimal M.Q., each parameter was selected as to enhance mixing. A final simulation was run with an angular velocity of $\omega=300 \mathrm{rad} / \mathrm{s}$ and a perpendicular length ratio of $\mathrm{b} / \mathrm{c}=6$ and a dual parallel channel structure. A mixing of $93.6 \%$ was reached. For even better mixing qualities, multi-channel structures could be used in series.

\section{5-Conclusion}

In this paper the effect of various geometric parameters and angular velocity on fluid mixing was observed in a centrifugal microfluidic micromixer. It was found that mixing is enhanced with increasing angular velocity. Increasing the angular velocity has the same effect within all cross sections and channel types. Thus, increasing the angular velocity of the rotating LOCD to a feasible value, is a proper means of enhancing the M.Q.. The inlet angle was found not to have a significant effect on the mixing process for inlet angles between 40 and 180 degrees which cover the entire feasible range for an LOCD design. The perpendicular length ratio has an optimal value of $b / c=6$ for which the best mixing occurs. Above that, increasing the $b / c$ ratio doesn't significantly increase the M.Q. It was also found that adding another microchannel in series significantly increases the M.Q. in a dual channel structure compared to a single channel structure. The orientation of the second channel does not affect the mixing significantly. Since the mixer length and dimensions are rather small compared to the CD diameter, M.Q. could be enhanced by considering additional channels in series. In another observation, the microchannel cross section was found to affect the flow pattern. Secondary flows, being a powerful mixing enhancer, were created best in channels with small aspect ratios. As for the cross-sectional profiles, regardless of fabrication concerns, the cross-sectional profiles which result in maximum M.Q.s were found to be the triangular, circular and rectangular profile; respectively. As for a proof of concept, a final simulation was run with optimal parameters in which an M.Q. of 93.6\% was obtained. Hence, the LOCD could serve as a mixing platform with desirable performance.

\section{Acknowledgement:}

The authors would like to thank Iran National Science Foundation: INSF and Sharif University of Technology grant for supporting this study.

\section{6-References}

[1] D. Figeys and D. Pinto, "Lab-on-a-chip: a revolution in biological and medical sciences," Analytical Chemistry, vol. 72, pp. 330 A-335 A, 2000.

[2] S. Haeberle, T. Brenner, H. P. Schlosser, R. Zengerle, and J. Ducrée, "Centrifugal micromixery," Chemical engineering \& technology, vol. 28, pp. 613-616, 2005.

[3] C. D. Chin, V. Linder, and S. K. Sia, "Lab-on-a-chip devices for global health: Past studies and future opportunities," Lab on a Chip, vol. 7, pp. 41-57, 2007.

[4] S.-P. Ryu, J.-Y. Park, and S.-Y. Han, "Optimum design of an active micro-mixer using successive Kriging method," International Journal of Precision Engineering and Manufacturing, vol. 12, pp. 849-855, 2011. 
[5] S.-J. Lee and B.-K. Choi, "The artificial glomerulus design using diffusion in microchannels," International Journal of Precision Engineering and Manufacturing, vol. 13, pp. 307-310, 2012.

[6] V. Srinivasan, V. K. Pamula, and R. B. Fair, "An integrated digital microfluidic lab-on-a-chip for clinical diagnostics on human physiological fluids," Lab on a Chip, vol. 4, pp. 310-315, 2004.

[7] R. B. Fair, "Digital microfluidics: is a true lab-on-a-chip possible?," Microfluidics and Nanofluidics, vol. 3, pp. 245-281, 2007.

[8] B. S. Lee, Y. U. Lee, H.-S. Kim, T.-H. Kim, J. Park, J.-G. Lee, et al., "Fully integrated lab-on-a-disc for simultaneous analysis of biochemistry and immunoassay from whole blood," Lab on a Chip, vol. 11, pp. 70-78, 2011.

[9] D. S. Kim, I. H. Lee, T. H. Kwon, and D.-W. Cho, "A barrier embedded Kenics micromixer," Journal of Micromechanics and Microengineering, vol. 14, p. 1294, 2004.

[10] D. S. Kim, S. W. Lee, T. H. Kwon, and S. S. Lee, "A barrier embedded chaotic micromixer," Journal of micromechanics and microengineering, vol. 14, p. 798, 2004.

[11] S. Lai, S. Wang, J. Luo, L. J. Lee, S.-T. Yang, and M. J. Madou, "Design of a compact disk-like microfluidic platform for enzyme-linked immunosorbent assay," Analytical chemistry, vol. 76, pp. 1832-1837, 2004.

[12] G. Jia, K.-S. Ma, J. Kim, J. V. Zoval, R. Peytavi, M. G. Bergeron, et al., "Dynamic automated DNA hybridization on a CD (compact disc) fluidic platform," Sensors and Actuators B: Chemical, vol. 114, pp. 173-181, 2006.

[13] M. J. Madou, L. J. Lee, S. Daunert, S. Lai, and C.-H. Shih, "Design and fabrication of CD-like microfluidic platforms for diagnostics: microfluidic functions," Biomedical Microdevices, vol. 3, pp. 245-254, 2001.

[14] Z. Noroozi, H. Kido, M. Micic, H. Pan, C. Bartolome, M. Princevac, et al., "Reciprocating flowbased centrifugal microfluidics mixer," Review of Scientific Instruments, vol. 80, p. 075102, 2009.

[15] S. Chakraborty, Microfluidics and microscale transport processes: CRC Press, 2012.

[16] Y. Wang, J. Zhe, B. T. Chung, and P. Dutta, "A rapid magnetic particle driven micromixer," Microfluidics and Nanofluidics, vol. 4, pp. 375-389, 2008.

[17] H.-Y. Wu and C.-H. Liu, "A novel electrokinetic micromixer," Sensors and Actuators A: Physical, vol. 118, pp. 107-115, 2005.

[18] T. Tofteberg, M. Skolimowski, E. Andreassen, and O. Geschke, "A novel passive micromixer: lamination in a planar channel system," Microfluidics and Nanofluidics, vol. 8, pp. 209-215, 2010.

[19] V. Hessel, H. Löwe, and F. Schönfeld, "Micromixers-a review on passive and active mixing principles," Chemical Engineering Science, vol. 60, pp. 2479-2501, 2005.

[20] A. D. Stroock, S. K. Dertinger, A. Ajdari, I. Mezić, H. A. Stone, and G. M. Whitesides, "Chaotic mixer for microchannels," Science, vol. 295, pp. 647-651, 2002.

[21] M. Grumann, A. Geipel, L. Riegger, R. Zengerle, and J. Ducree, "Batch-mode mixing on centrifugal microfluidic platforms (vol 5, pg 560, 2005)," LAB ON A CHIP, vol. 5, pp. 1184-1184, 2005.

[22] D. C. Duffy, H. L. Gillis, J. Lin, N. F. Sheppard, and G. J. Kellogg, "Microfabricated centrifugal microfluidic systems: characterization and multiple enzymatic assays," Analytical Chemistry, vol. 71, pp. 4669-4678, 1999.

[23] J. V. Zoval and M. J. Madou, "Centrifuge-based fluidic platforms," Proceedings of the IEEE, vol. 92, pp. 140-153, 2004.

[24] L. G. Puckett, E. Dikici, S. Lai, M. Madou, L. G. Bachas, and S. Daunert, "Investigation into the applicability of the centrifugal microfluidics platform for the development of protein-ligand binding assays incorporating enhanced green fluorescent protein as a fluorescent reporter," Analytical chemistry, vol. 76, pp. 7263-7268, 2004. 
[25] M. La, S. J. Park, H. W. Kim, J. J. Park, K. T. Ahn, S. M. Ryew, et al., "A centrifugal force-based serpentine micromixer (CSM) on a plastic lab-on-a-disk for biochemical assays," Microfluidics and nanofluidics, vol. 15, pp. 87-98, 2013.

[26] L. Falk and J.-M. Commenge, "Performance comparison of micromixers," Chemical Engineering Science, vol. 65, pp. 405-411, 2010.

[27] W. Ehrfeld, K. Golbig, V. Hessel, H. Löwe, and T. Richter, "Characterization of mixing in micromixers by a test reaction: single mixing units and mixer arrays," Industrial \& Engineering Chemistry Research, vol. 38, pp. 1075-1082, 1999.

[28] J. Aubin, D. F. Fletcher, J. Bertrand, and C. Xuereb, "Characterization of the mixing quality in micromixers," Chemical Engineering \& Technology, vol. 26, pp. 1262-1270, 2003.

[29] J. Zalc, E. Szalai, F. Muzzio, and S. Jaffer, "Characterization of flow and mixing in an SMX static mixer," AIChE journal, vol. 48, pp. 427-436, 2002.

[30] P. Danckwerts, "The definition and measurement of some characteristics of mixtures," Applied Scientific Research, Section A, vol. 3, pp. 279-296, 1952.

[31] K. Malecha, L. J. Golonka, J. Bałdyga, M. Jasińska, and P. Sobieszuk, "Serpentine microfluidic mixer made in LTCC," Sensors and Actuators B: Chemical, vol. 143, pp. 400-413, 2009.

[32] P. Kundu and L. Cohen, "Fluid Mechanics, 638 pp," Academic, Calif, 1990.

\section{Captions:}

Fig.1 Schematic of the geometry of the micromixer

Fig. 2 various rectangular cross sections, dimensions in $\mu \mathrm{m}$

Fig.3 Different types of channel structures

Fig.4 the employed structured mesh with two magnified regions

Fig.5 (a) concentration contour for the validation simulation (b) quantitative comparison of the simulation results with experimental data by La et al.[25]

Fig.6 M.Q. for different perpendicular length ratios (b/c)

Fig.7 The velocity/concentration contour for dual parallel channel for $\omega=200 \mathrm{rad} / \mathrm{s}$

Fig. 8 The velocity/concentration contour for dual anti-parallel channel for $\omega=200 \mathrm{rad} / \mathrm{s}$

Fig.9 M.Q. vs. $\omega$ in the single channel

Fig.10 M.Q. vs. $\omega$ in the dual parallel channels

Fig.11 M.Q. vs. $\omega$ in the dual anti-parallel channels

Fig.12 M.Q. for various inlet angles

Fig.13 whole-channel (up) and middle-cross sectional (down) concentration contours $\left(\mathrm{mol} / \mathrm{m}^{3}\right.$ ) and their respective velocity vector field. (a) Vertical Triangular (b) Equilateral Triangular (c) Horizontal Triangular 
Fig.14 whole-channel (up) and middle-cross sectional (down) concentration contours $\left(\mathrm{mol} / \mathrm{m}^{3}\right.$ ) and their respective velocity vector field. (a) Vertical Elliptic (b) Circular Elliptic (c) Horizontal Elliptic

Fig.15 whole-channel (up) and middle-cross sectional (down) concentration contours $\left(\mathrm{mol} / \mathrm{m}^{3}\right.$ ) and their respective velocity vector field. (a) Vertical Rectangular (b) Square Rectangular (c) Horizontal Rectangular 

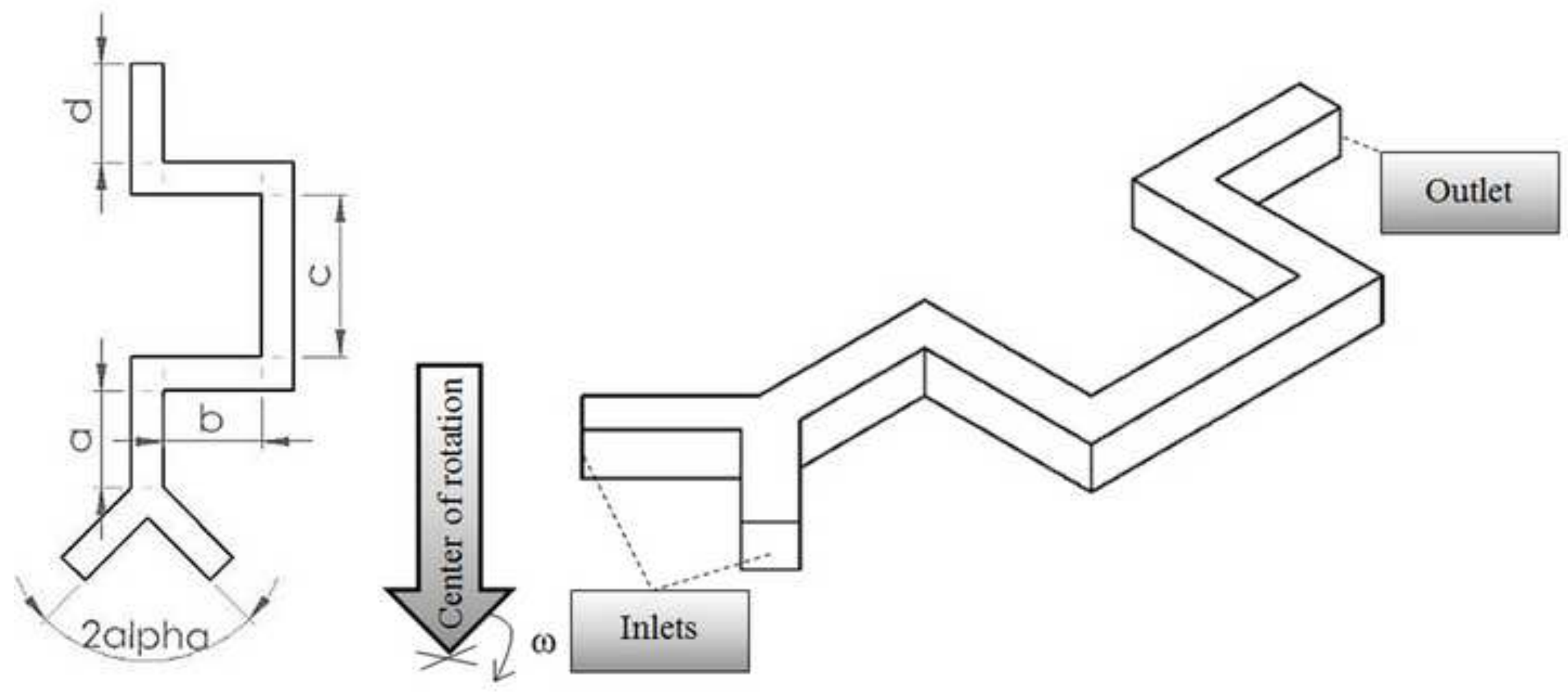


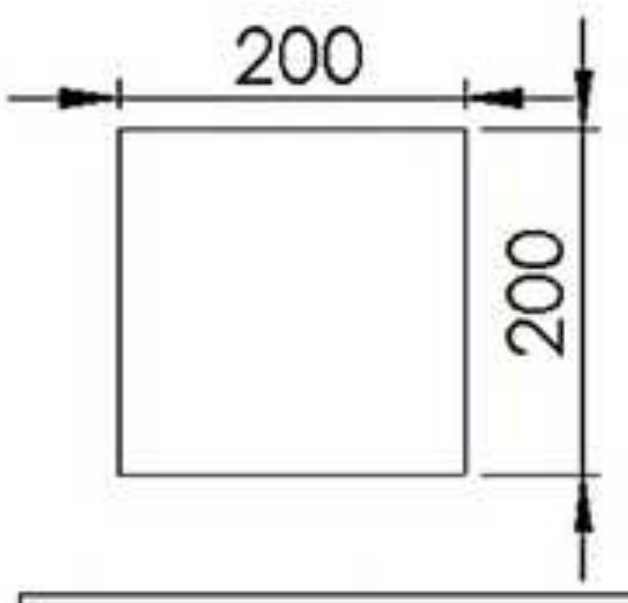

Square Cross section Aspect Ration 1
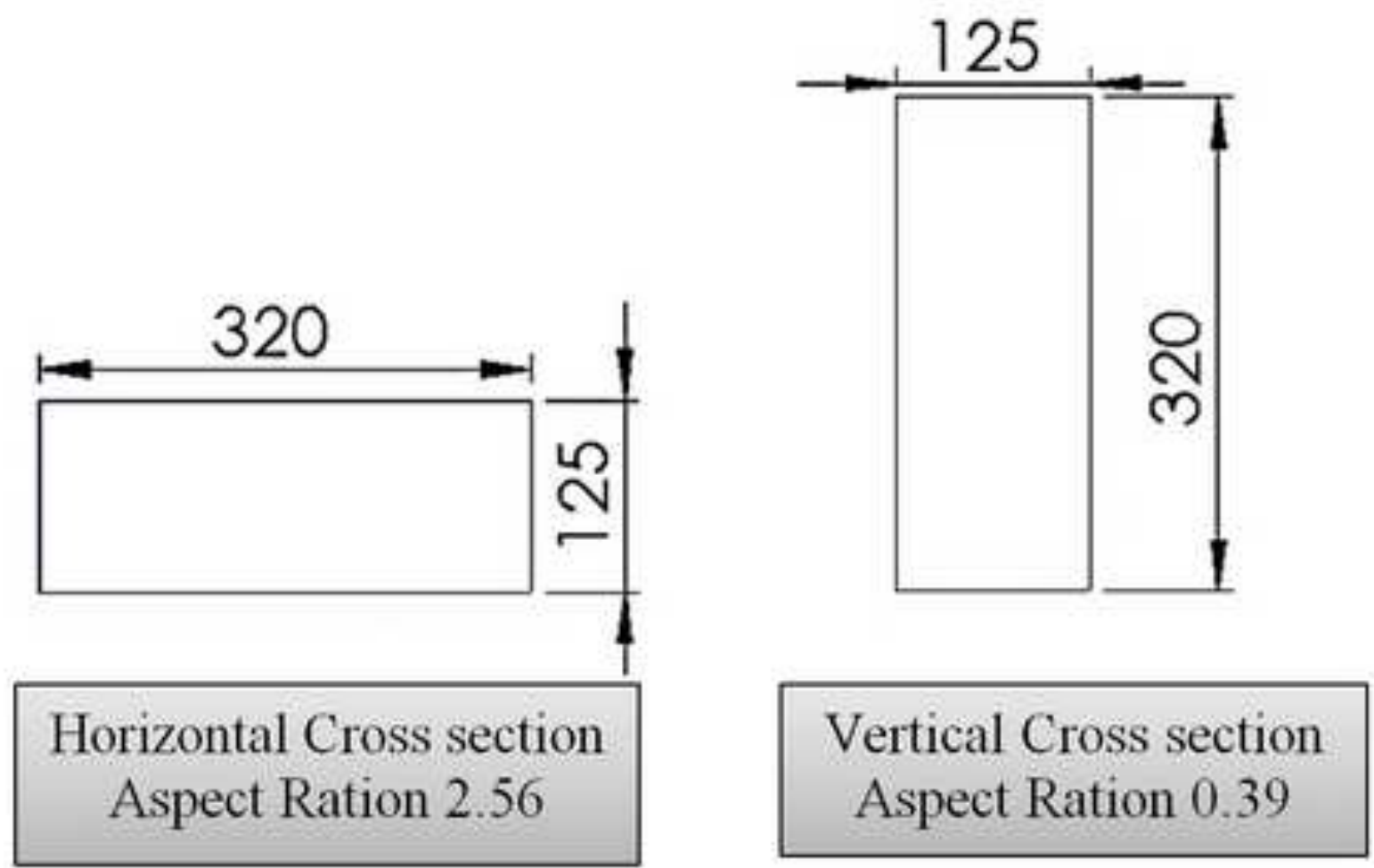

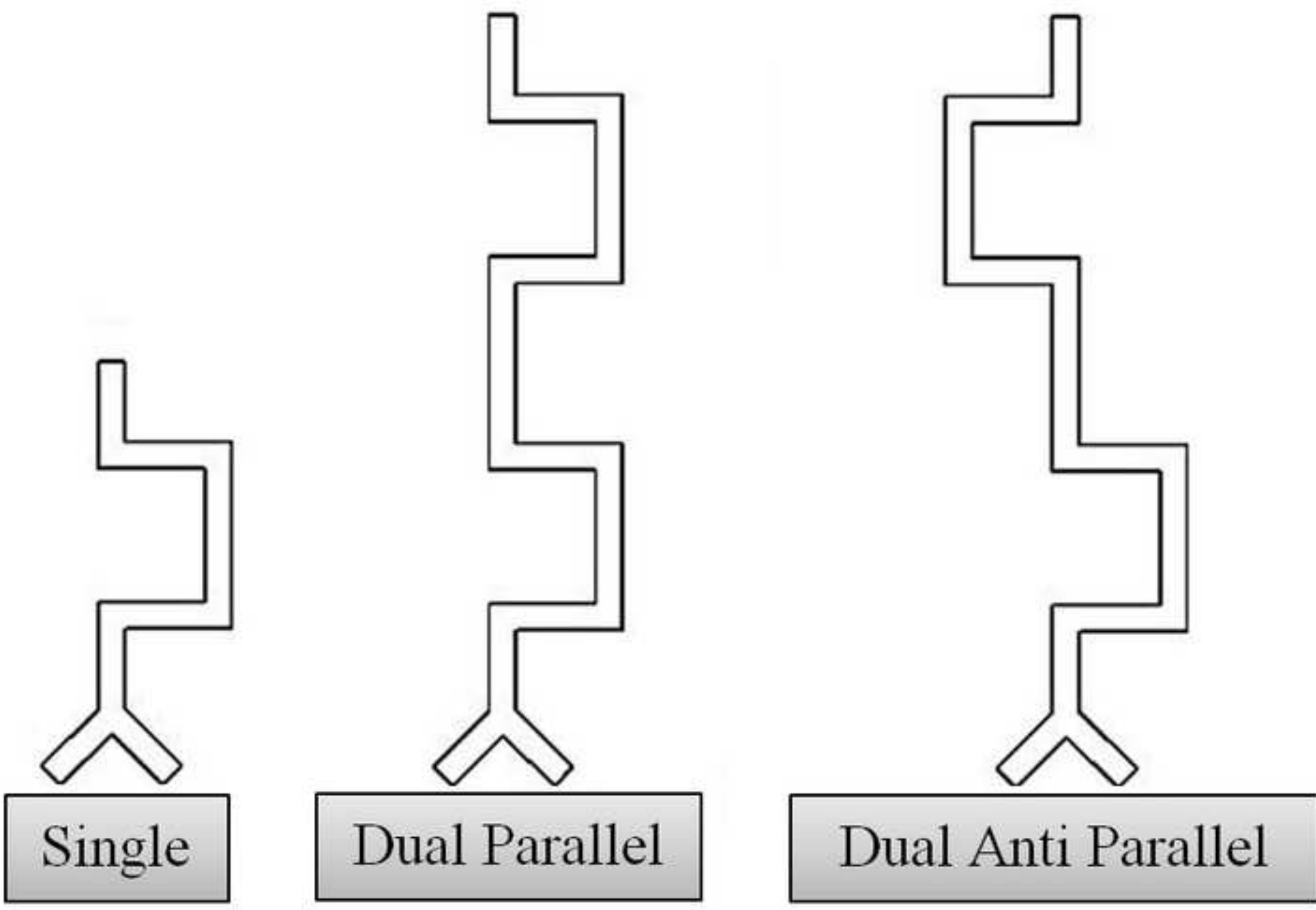

\section{Dual Anti Parallel}




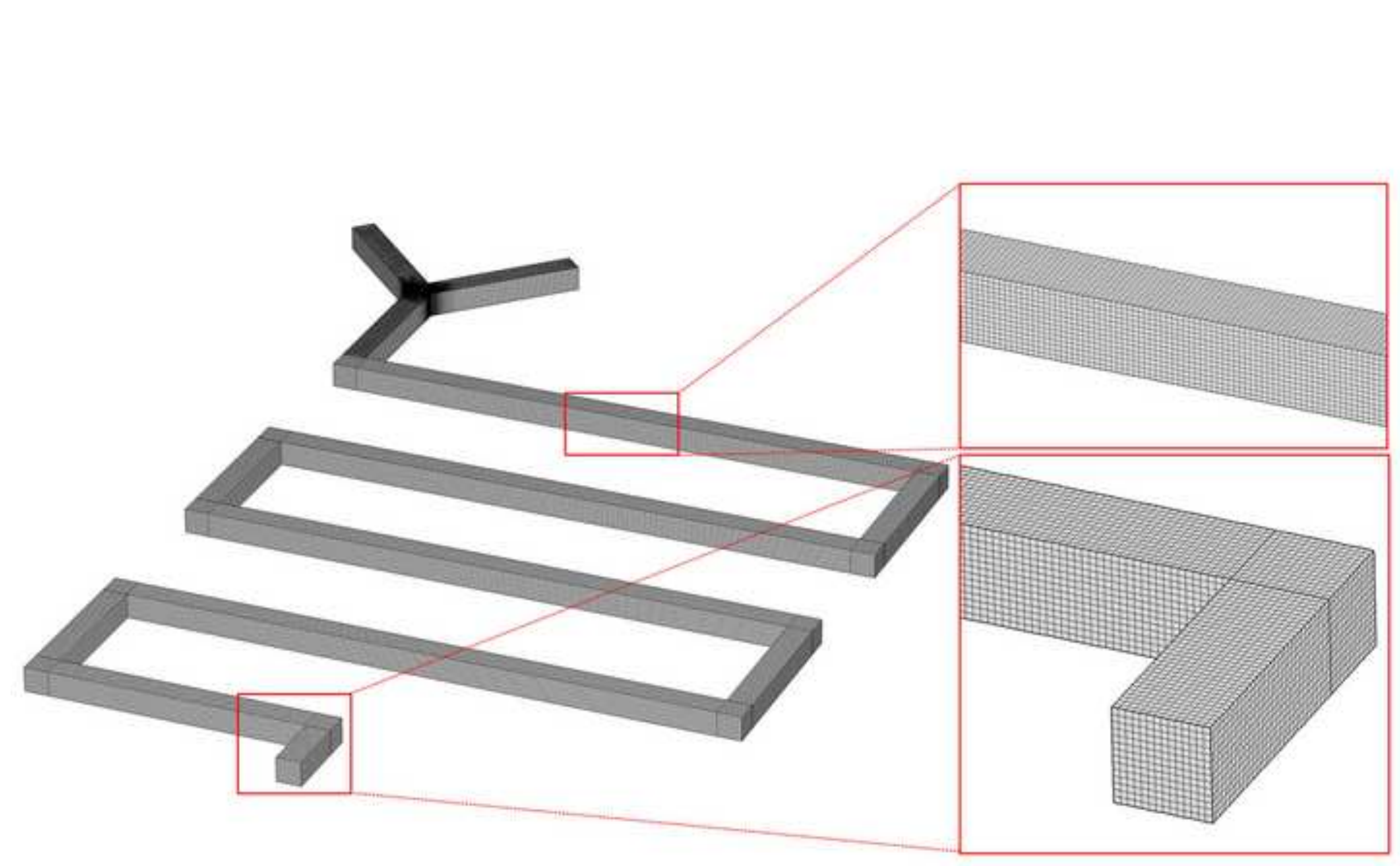




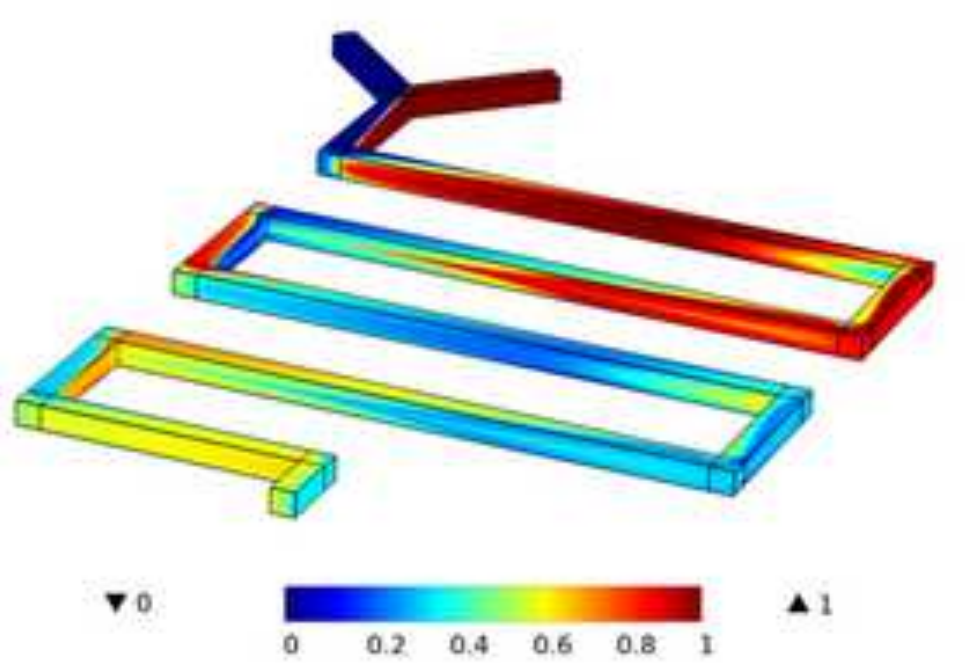

(a)

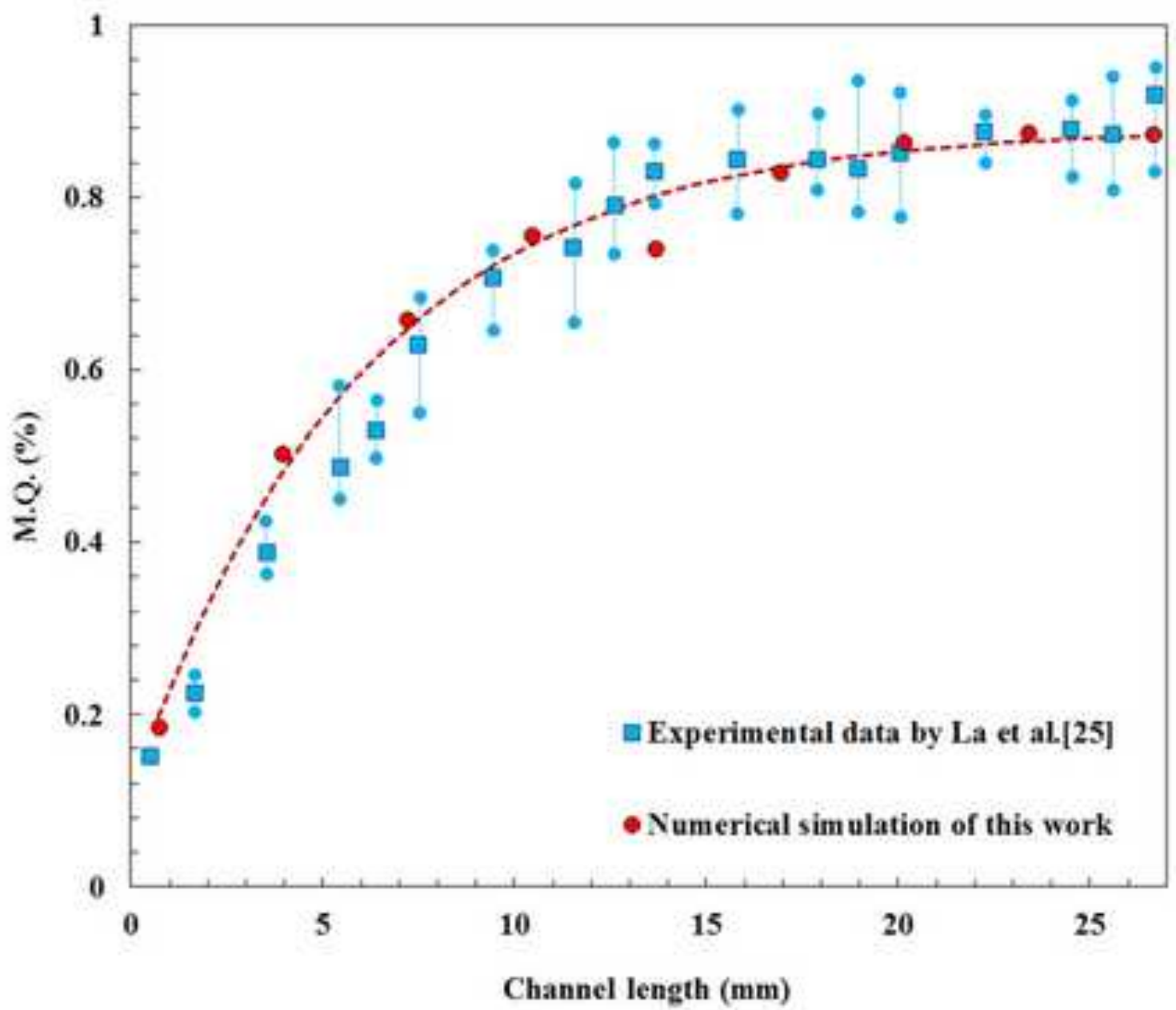

(b) 


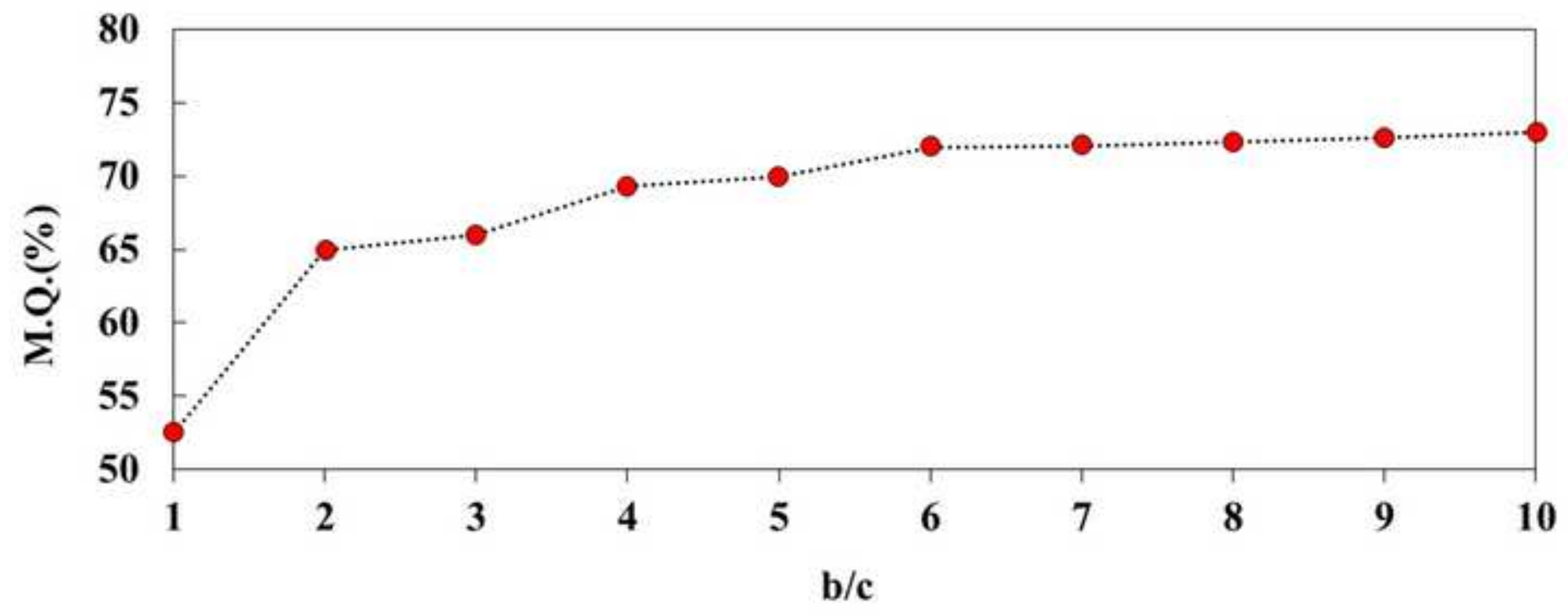



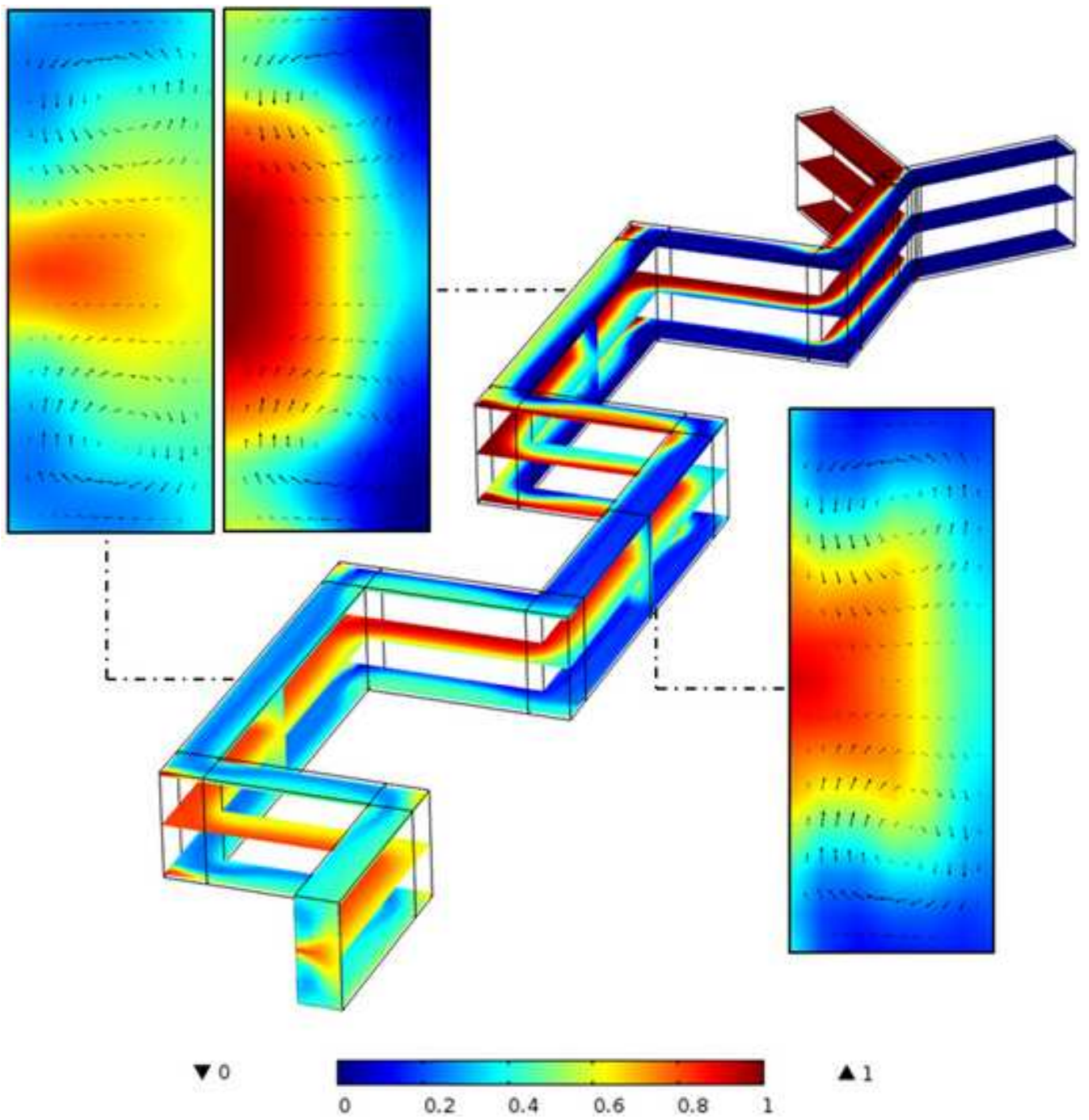

A 1

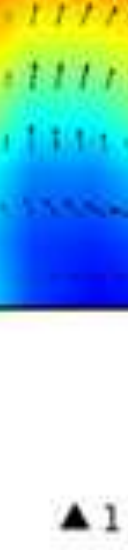



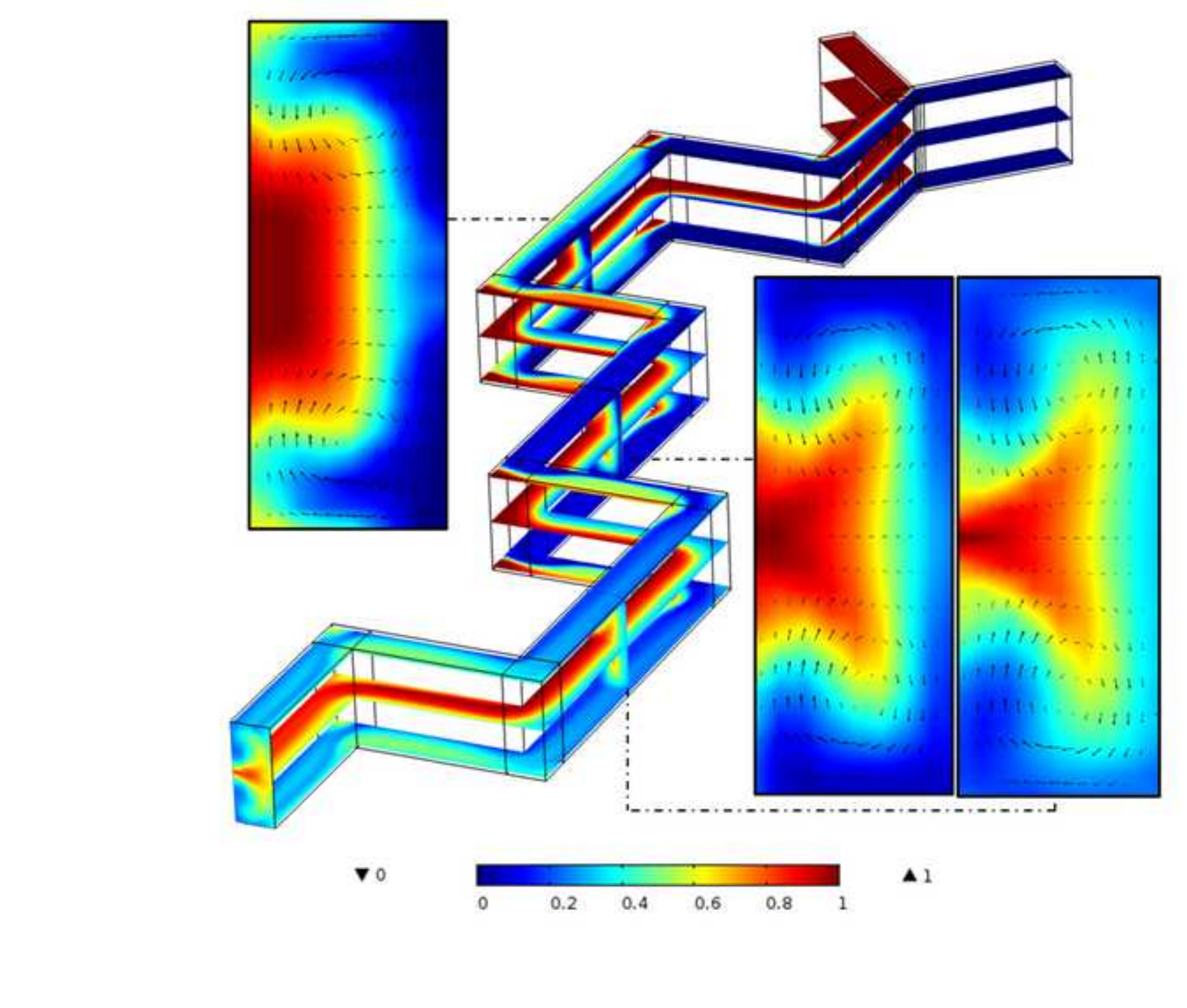

8

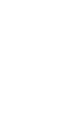

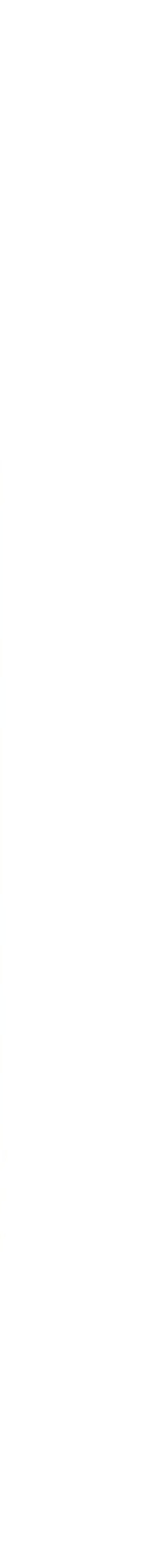

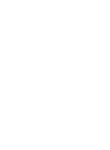




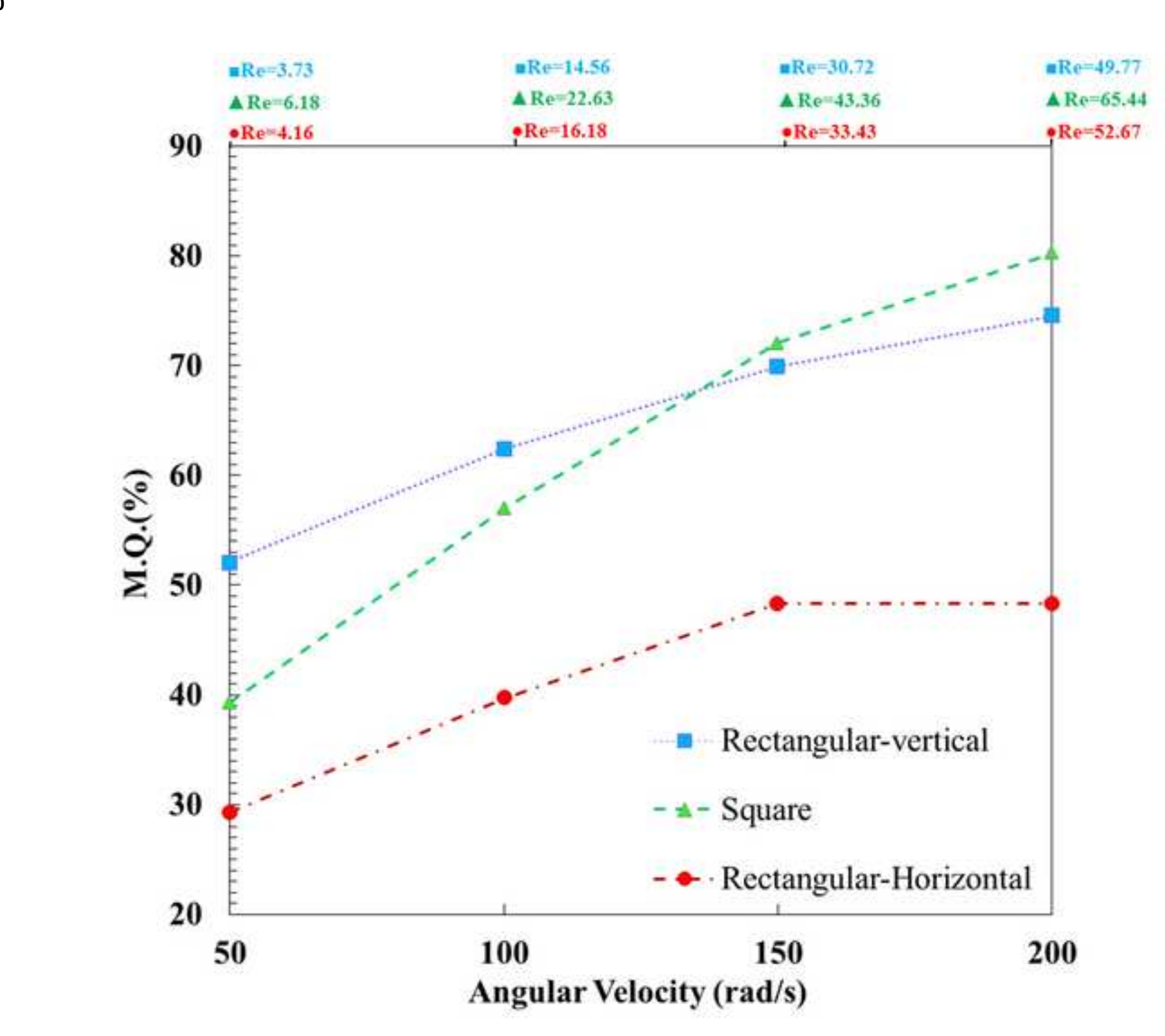




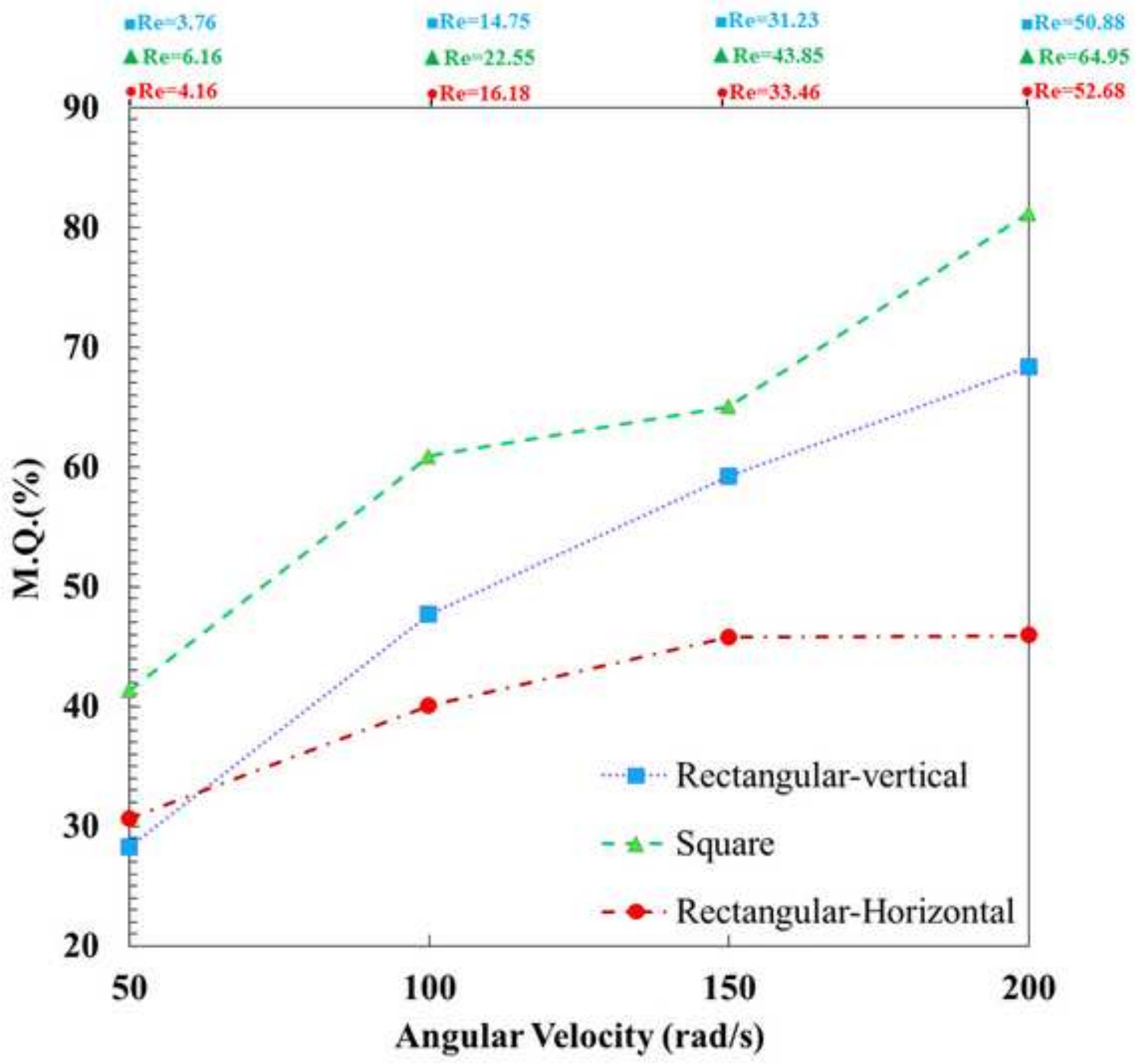




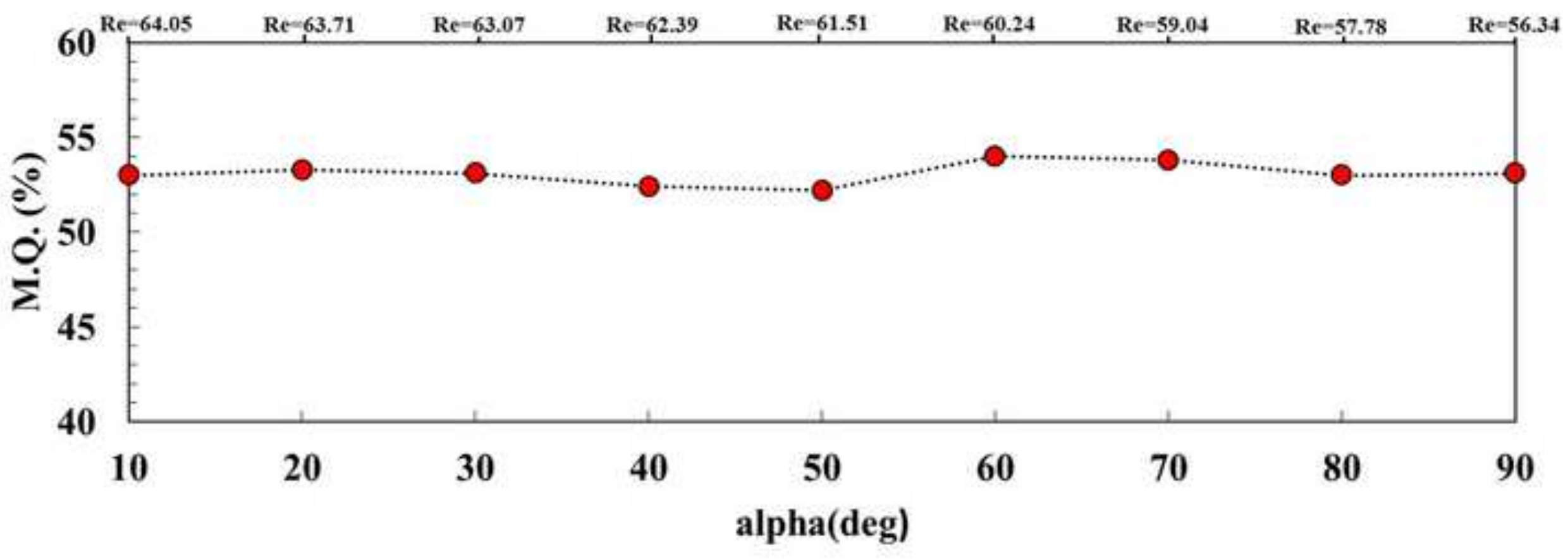




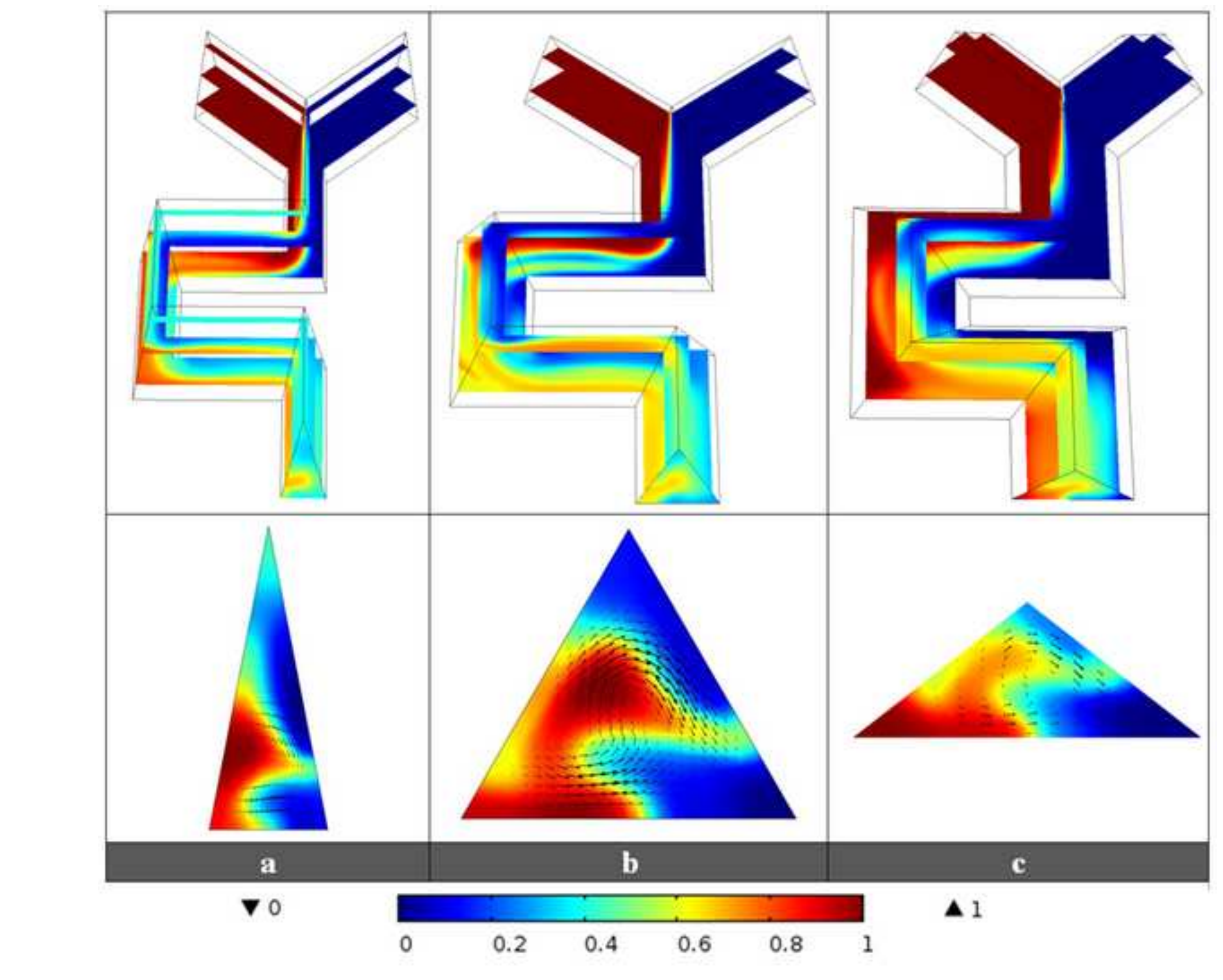

Figure 13

3

.

Figure

(2)
.

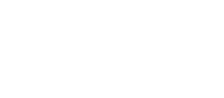
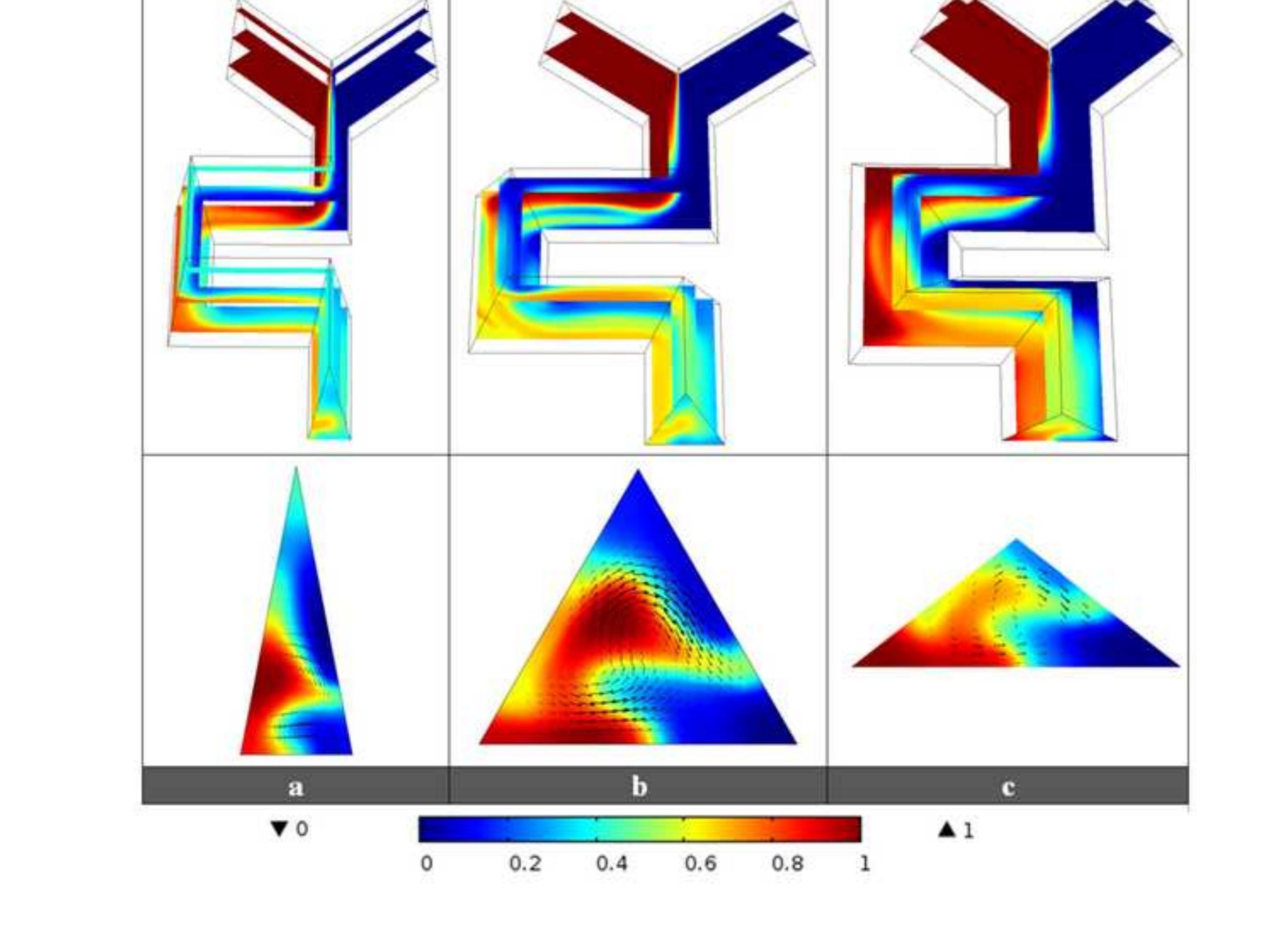

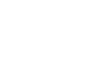
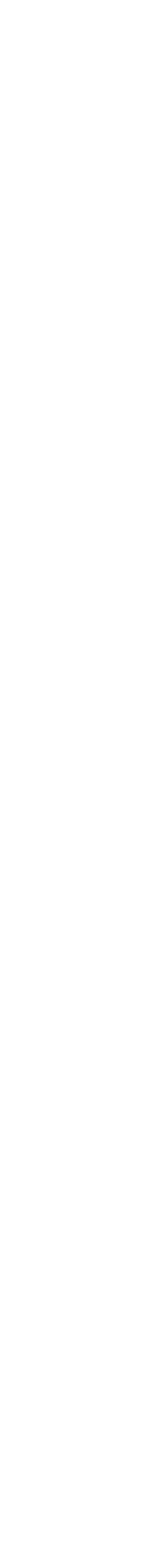


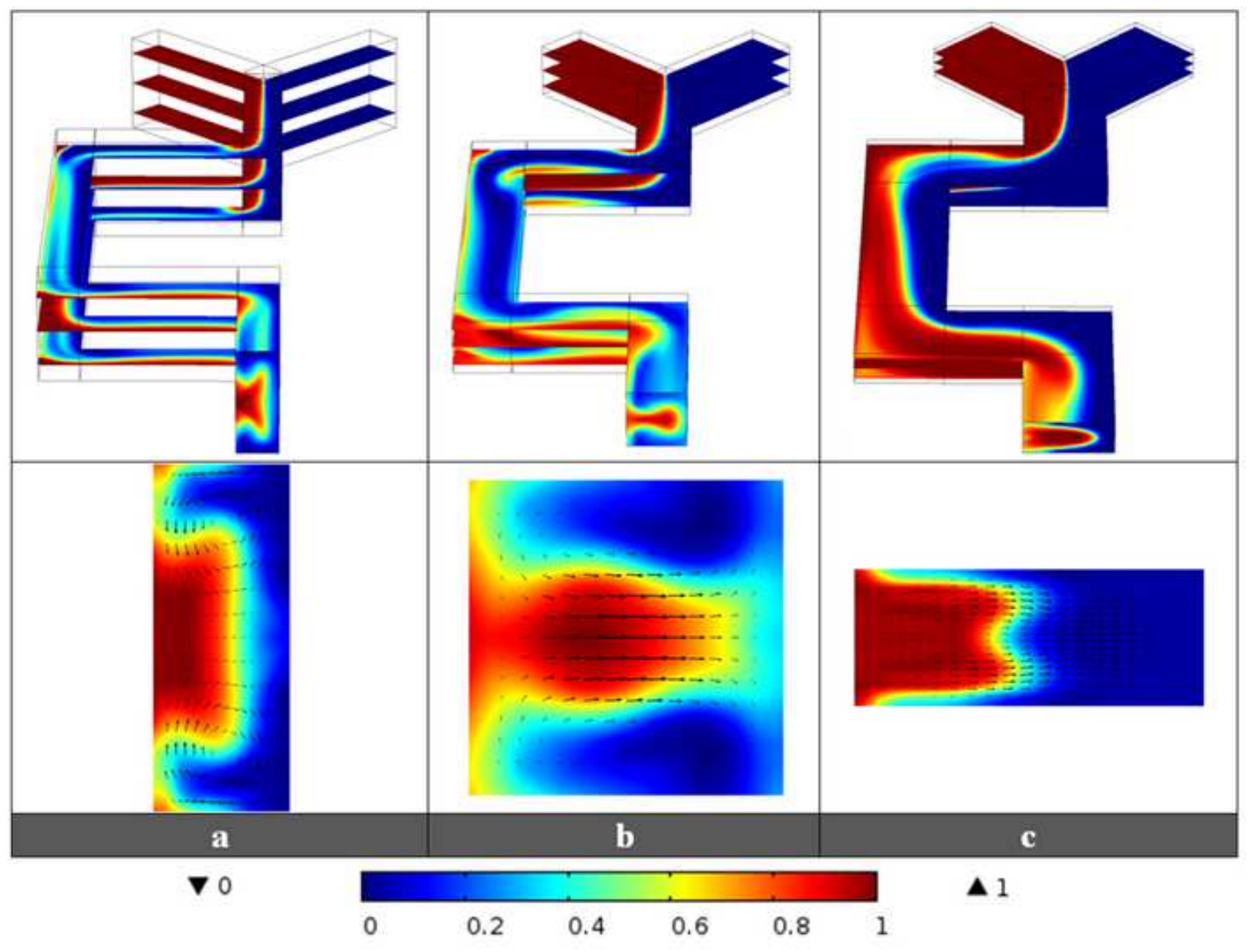

